\title{
XVI.
}

\section{Wurde Theodosius von Gratian zunächst zum ma- gister militum und erst nach einem siege über die Sarmaten zum kaiser ernannt?}

Im neunten capitel seines Panegyricus auf Theodosius den Grossen ${ }^{1}$ ) schildert Pacatus einen aufenthalt des Theodosius in Spanien, welcher bisher betrachtet wurde als die zeit der freiwilligen verbannung, durch welche Theodosius dem schicksale seines vaters zu entgehen suchte, der 375 durch Gratian getödtet wurde. R. Nitsche, Gothenkrieg (Altenburg, programm 1871) p. 11 f. n. 7 bestreitet diese auffassung und meint, es müsse der hier geschilderte aufenthalt vor 374 gelegt werden, weil der sieg, den Theodosius nach Pacatus c. $\mathbf{X}$ über die Sarmaten gewann, nachdem er kaum aus Spanien an die Donau zurückgekehrt war, in das jahr 374 falle.

Damals war Theodosius dux von Mösien und gewann einen sieg über plündernde Sarmaten, der ihm hohen ruhm eintrug, dieser sieg könnte also von Pacatus gemeint sein, störend ist nur, dass Theodosius vor diesem siege längere zeit in Spanien friedlichen studien obgelegen haben soll. Nicht nur, dass wir bei denjenigen schriftstellern, welche bestimmt von dem siege des jahres 374 sprechen, hiervon nichts hören, es passt eine solche annahme auch nicht besonders zu dem, was wir von dem leben des Theodosius wissen. Theodosius war 37429 jahr alt und es ist nicht

1) Der Panegyricus wurde zu Rom im senat gesprochen 389 (Tillemont Histoire des Empereurs V, 303): Pacatus, ein gallischer rhetor, war von seiner provinz nach Rom geschickt, um den Theodosius wegen der unterdrückung des aufstandes des Maximus zu beglückwünschen. Ich citire nach der ausgabe in Panaegyrici Veteres ed $J$. de la Baune ed. altera Venetiis 1728. 
sehr wahrscheinlich, dass er seine kriegerische laufbahn so früh schon durch eine längere freiwillige musse unterbrochen habe.

Doch würden solche zweifel wenig bedeuten, wenn nicht Theodoret in seiner kirchengeschichte ${ }^{2}$ ) erzählte, Theodosius sei nach dem unglück von Adrianopel von Gratian aus Spanien herbeigerufen und zum magister militum ernannt. Theodosius schlug die barbaren und Gratian erhob ihn aus freude hierüber zum kaiser des ostens. Es ist natürlich in diesem siege den kampf gegen die Sarmaten bei Pacatus wieder zu finden, da beide unmittelbar auf einen aufenthalt in Spanien folgen. Schon früher ist jedoch diese erzählung angezweifelt, und Nitsche verwirft sie mit entschiedenheit, weil die übrigen quellen den sieg des Theodosius nicht erwähnten, weil vor allem Themistius in der rede davon schweige, mit welcher er als gesandter der hauptstadt Constantinopel den Theodosius bei seiner erhebung zum Augustus beglückwünschte, und weil endlich drittens einige worte des Pacatus auf eine frühere zeit zurückwiesen. Von diesen drei gründen wird der erste am schluss der untersuchung seine erledigung finden, von weit grösserem gewicht sind zwei und drei. Nitsche fasst nr. 2 in folgender weise. Themistius hätte den sieg erwähnen müssen. „Dies thut er aber nicht nur nicht, sondern er sagt sogar mit worten, welche an deutlichkeit nichts zu wünschen übrig lassen, dass Theodosius den Gothen noch keine feldschlacht geliefert habe:

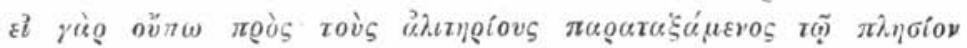

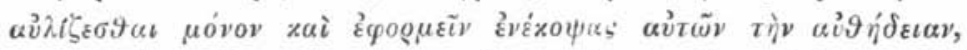

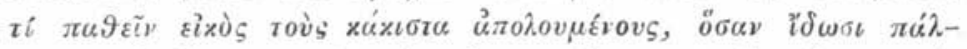

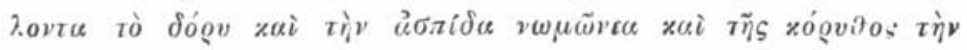

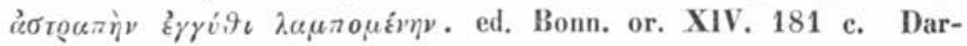
aus folgert Nitsche: der Sarmatensieg des Theodosius, den Pacatus erwähnt, ist kein anderer als der 374 errungene.

Allein Nitsche sagt selbst, dass jene stelle auf die Gothen gehe (kurz vorher steht der name $\Sigma_{x v} q \alpha \iota_{5}$ Gothen, weiche The-

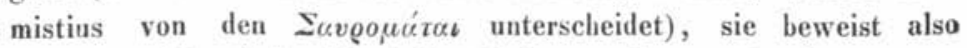
nur, dass Theodosius bis dahin (einige wochen nach seiner ernennung zum kaiser) gegen die seit dem siege von Adrianopel Thracien verwüstenden Gothen noch nicht gekämpft hatte. Nitsche

2) Theodoreti historia ecclesiastica l. V, 5. 6 ex editione Henr. Valesii, Mogunt. 1679 p. $204 \mathrm{f}$. 
geht zu weit, wenu er schliesst, dass Theodosius bis dahin überhaupt noch keinen sieg gewonnen haben könne, und dass deshalb auch der von Theodoret erwähnte kampf nothwendiger weise als eine erfindung angesehen werden müsse. Könnte Theodosius nicht über andere barbaren gesiegt haben, welche die noth der Römer zu einem einfall über die Donau benutzten? Freilich, wenn Theodoret auch darin recht hätte, dass dieser sieg dem Theodosius die krone eintrug, so würde Themistius dies mit unzweidentigen lobeserhebungen preisen, aber davon abgesehen, wäre es so undenkbar, dass Theodosius durch einen glücklichen angriff einen theil der Donaulande von den plünderungen befreite und dass die Constantinopolitaner sammt ihrem rhetor wenig darauf achteten, sondern nach wie vor vor den mächtigen Gothen zitterten, welche sie näher bedrängten? An barbaren, welche einen solchen einfall machen und also geschlagen werden konnten, fehite es nicht, noch 376 hatte Athanarich gerade Sarmaten aus ihren sitzen in Siebenbürgen gedräingt, die leicht noch ohne heimat umherstreifen mochiten.

Richter ${ }^{3}$ ) glaubt übrigens für einen solchen sieg des Theodosius uiber Sarmaten ein unmittelbares zeugniss bei Themistius gefunden zu haben. Die stelle lautet: ,mit recht wählte Gratian denjenigen, den die noth der zeit vorher bezeichnet hatte. So rief auch den Thebaner Epaminondas, der als soldat im glied stand, die gefalır an die feldherrnstelle. Von dem tage an forderten dich die Römer zum kaiser, an dem du die Sarmaten, welche die lande an der Donau plündernd durchzogen allein vertriebst und noch dazu mit einer kleinen und nicht ausgewählten schaar".

Hier ist schwer zu entscheiden, ob Themistius den sieg von 374 oder, wenn er kein fabel ist, den andern von $378 \mathrm{im}$ sinne hat. Der sieg von 374 ist bei Ammian 29,6 und bei Zosimus IV, 16 überliefert. Ammian spricht mit grosser anerkennung von der that des: invenis prima etiam tum lanugine, Princeps postea perspectissimus, und Zosimus knïpft an seine kurze erzahllung die

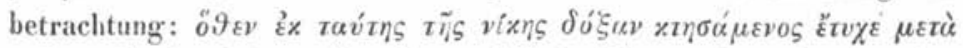

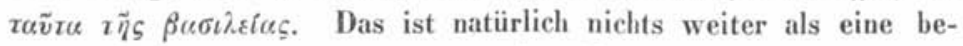
trachtung wie sie angestellt zu werden pflegt über die ersten thaten, durch welche ein bedeutender mann seine ruhmvolle laufbahn er-

3) Das weströmische reich p. $691 \mathrm{f}$. 
öffnet, aber sie trifft wahrscheinlich das richtige. Es ist nicht unwahrscheinlich, dass gerade das tapfere verhalten in Mösien 374 den Gratian bewog, den Theodosius aus seiner verbannung zu rufen.

Da nun andererseits von dem siege des jahres 378 nur unbestimmte kunde $\mathrm{zu}$ uns gekommen ist, so darf man wohl sagen: der sieg von 374 war auch bei den zeitgenossen der bekanntere. Wenn Themistius daher ganz allgemein einen Sarmatensieg des Theodosius erwähnt, so wird man zunächst vermuthen, dass der von 374 gemeint sei. Umgekehrt fällt die entscheidung aus, wenn die betrachtung ausgeht von den worten: „seit dem siege über die Sarmaten forderten dich die Römer zum kaiser" (Themist. ed. Bonn.

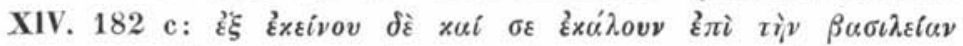

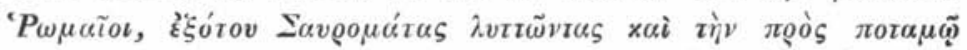

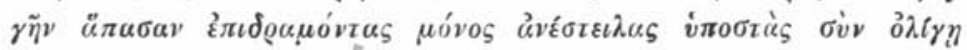

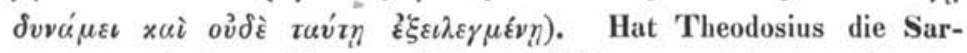
maten zweimal geschlagen, 374 und 378 , so wird man hier zunächst vermuthen, dass der sieg von 378 gemeint sei. Der consensus omnium ist freilich in jedem falle eine rhetorische übertreibung, allein es hat doch einen guten sinn, wenn die Römer den Theodosius im jahre 378 zum nachfolger des Valens wünschen, als sein name durch den plötzlichen und wie es scheint schuldlosen sturz seines gleichnamigen heldenhaften vaters und durch die verbannung nach Spanien in aller munde war, zumal wenn er diese erinnerung durch einen sieg belebte, der zwar nicht über den hauptfeind, die gefürchteten Gothen, errungen war, aber doch den verzweifelnden Römern als der anfang einer wendung zum besseren erscheinen durfte. Im j. 374 dagegen lebte Valens noch, ein tapferer mann und unermüdlicher krieger und wenn die Römer eines andern bedurft hätten, so wäre ihre wahl sicher eher auf Theodosius den vater gefallen, der damals in der blüthe seines ruhmes stand, als auf den sohn, dem der erste flaum um den mund spielte und bis zu jenem commando in Mösien stets unter des vaters leitung gekämpft zu haben scheint.

Für 374 wäre demnach jene bemerkung des Themistius eine geschmacklosigkeit, und wenn wir ihm solche auch in vollem maasse zutrauen,' so spricht die stelle zunächst doch für 378 . Ebenso passt auf 378 besser der schluss jener stelle, Theodosius habe den sieg gewonnen mit wenigen und nicht gerade ausge- 
wählten schaaren. Für 378 als Theodosius plötzlich von Spanien ankam und seine armee aus den trümmern der alten neu bilden musste, hat dies seine entschuldigung, der regelmässige beamte von Mösien musste aber doch seine truppen in ordnung haben, für ihn wäre es ein zweifelhaftes lob. Es lassen sich auch hier freilich eine reihe von möglichkeiten denken, welche dies erklärten aber leichter giebt sich diese erklärung für 378. Sowohl auf

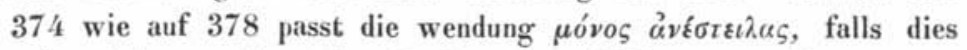
$\mu o ́ v o \varsigma$ heissen soll: wälırend alle andere feldherrn geschlagen wurden oder sich in die festen plätze zurückzogen. Und wenn dies uóvos seine erklärung durch das folgende: mit einer unzureichenden macht finden und also heissen soll: deine klugheit und kühnheit gab den ausschlag, so haben wir eine höfliche wendung, die ebenfalls für beide jahre passt. Wenn wir die grïnde wägen, die sich so für die eine oder andere deutung geltend machen lassen, so werden manche dem ersten das meiste gewicht zusprechen und sagen: da der sieg von 374 der bekanntere gewesen zu sein scheint, so ist es wahrscheinlich, dass Themistius diesen sieg meinte. Wenig trägt es aus, dass seine schmeichelei dann fast sinnlos erscheint. Man kann so urtheilen, aber zu entschiedenem resultate ist hier nicht zu kommen und wir müssen uns deshalb zu dem Pacatus wenden, da eine andere stelle des Themistius, $\mathbf{X V}$, 189, welche einen sieg des Theodosius über Sarmaten erwähnt, und der umstand, dass Gratian von Ausonius Sarmaticus genannt wird ${ }^{4}$ ), nichts entscheiden können. Schon jener erste sieg von 374 konnte den dichter veranlassen dem Gratian, der damals bereits bei lebzeiten seines vaters den kaisertitel führte, diesen namen beizulegen, und auch sonst wird es gewiss nicht an kleinen kämpfen mit den Sarmaten gefehlt haben; mehr bedarf es aber für einen panegyristen nicht, um solche beinamen zu verleihen. Pacatus schildert den spanischen aufenthalt des Theodosius mit einer summe rhetorischer wendungen, welche nur wenig thatsächliches enthalten und es auch bei genauer kunde von dem leben des Theodosius während seiner verbannung schwerlich gestatteten, den be-

4) Richter 1. c. 692 führt dies als beweisstelle an für den sieg von 378. Sie steht zwar am schluss der note, als der beweis nach Richters meinung schon geführt ist, doch hätte der sehr beschränkte werth dieses zeugnisses ausdrücklich hervorgehoben werden sollen. 
stimmten nachweis zu führen, dass diese zeit gemeint sei oder nicht. Allein so viel darf man doch wohl sagen: die-schilderung macht den eindruck, als gehe sie auf einen dauernden aufenthalt. Dies spricht gegen Nitsche's vermuthung, da wir schon oben sahen, dass es an und für sich nicht wahrscheinlich ist, dass Theodosius vor 374 seine kriegerische laufbahn durch eine längere friedliche musse unterbrochen hat.

Auch kämpfte Theodosius in Mösien 374 so viel wir wissen zum ersten male als selbständiger anführer und daher würde sowohl der ausdruck c. $\mathbf{X}$ arma emerita suspenderas als auch die ähnliche wendung c. IX, weil du schon ein meister der kriegskunst warst" (quia iam ad plenum bellicis artibus abundabas) wenig passen auf die zeit vor 374. Freilich sagen die rhetoren oft, was wenig passt, und ein entscheidendes gewicht ist hierauf nicht zu legen, entscheidend ist digegen, dass Pacatus nicht undeutlich erkennen lässt, der aufenthalt in Spanien sei kein freiwilliger gewesen, sondern durch ein geschick erzwungen. „Wie verborgen, heisst es im eingange des c. $\mathbf{I X}{ }^{5}$ ), sind doch stets die wege des schicksals! Wer, frage ich, lätte nicht deinen weggang aus dem lager als ein unglück für den staat angesehen? Allein das geschick wollte den künftigen kaiser vorbereiten und wollte deshalb, dass du eine zeitlang als privatmann lebtest, damit du, der in den künsten des kriegs schon meister war, auch die bürgerlichen verhältnisse kennen lerntest.

Es ist nicht wahrscheinlich, dass Pacatus so von einem anfenthalte sprechen würde, den Theodosius freiwillig gewählt hätte. Würde der lobredner nicht vielmehr den freien blick des künftigen imperator gepriesen haben, der als lorbeerbekränzter jüngling die wichtigkeit der bürgerlichen geschäfte nicht verkannte und seine ruhmeslaufbahn unterbrach, um in unscheinbare verhältnisse zurückzutreten, ja noch mehr, um in denselben bescheiden zu lernen?

Nitsche stellt diese erwägungen nicht an, er beruft sich für seine annahme auf die worte: ut iam $t u m$ posset intelligi alios

5) Pac. c. IX: Quam tecta sunt semper consilia fortunae! Quis, quaeso, tum publicis rebus non pulasset, ininicum turm illum a statione castrensi ad quietem receptum? Eninecro illa futurum principem comens, idcirco paulisper voluit esse privatum ut quia iam ad plenum bellicis artibus abundabas, usus civilis experiens sub otii tempore reddereris. 
imperatori pugnare, te tibi. Diese worte schliessen eine lange reihe von rhetorisclien wendungen, in denen Theodosius gepriesen wird, er habe als er bei seiner rückkehr aus Spanien an der Donau gegen die Sarmaten zu felde lag, die besclıwerden des gemeinen soldaten getheilt. Nitsche sagt p. 12, n. 7: ,mun kann aus diesen worten iam tum ersehen, dass hier nicht von ereignissen die rede ist, welche wenige tage oder wochen der ernennung des Theodosius zum kaiser vorausgehen". Ich kann dies nicht einsehen, ich finde, dass der nachdruck einzig darauf liegt, dass sich Theodorich so eifrig erwies, bevor er kaiser war, auf die länge der zwischenzeit kommt es nicht an. Daneben macht Nitsche geltend, dass Pacatus jene verbannung nicht einmal gut habe schildern können, ,er übergeht in seiner festlichen lobrede diese auch in der erinnerung unangenehmen ereignisse klüglich mit stillschweigen". Allein, wenn Pacatus dies beabsiclitigte, so durfte er Spanien nicht nennen, oder wenn Theodosius sich wirklich schon vor $374 \mathbf{z u}$ einer längeren musse nach Spanien zurückgezogen liätte, und Pacatus wollte dies nicht übergehen und doch die erinnerung an die verbannung vermeiden: so musste er jahr und tag dieses aufenthaltes genau angeben. Wie die worte jetzt lauten, haben hörer und leser gewiss an die verbannung gedacht, die lange zeit die gemüther lebhaft beschäftigt hatte und besonders seit Theodosius kaiser wurde. Auch kann Nitsche nicht sagen, diese zeitbestimmung sei für die zeitgenossen mit hinreichender sicherheit in der erwähnung des Sarmatensieges gegeben, da die fabel des Theodoret erst um 450 entstanden sei, denn jene erwähnung folgt erst im folgenden capitel, als die unliebsamen erinnerungen lizngst schon belebt waren. Mit einem gewissen scheine köunte man dagegen für Nitsche's ansicht folgende erwägung anstellen.

Pacatus erwähnt nur einen Sarmatensieg des Theodosius, wäre dies nicht der im jahre 374 erfochtene, so hätte Pacatus diesen vielgepriesenen sieg ganz übergangen und das wäre doch aufiallend. Allein die rhetoren übergehen vielfach das grosse und erwähnen das kleine, wenn es nur in ilıre antithetischen spielereien und schïlerhaften beispiele passt. Zudem giebt Pacatus überhaupt keine bestimmten einzelheiten weiter aus der kampfreichen jugendzeit des Theodosius, er erwahnt nicht einmal den namen Britanniens, wo der jüngling unter des vaters leitung kämpfte. 
So ist denn allem anschein nach unter dem aufenthalt in Spanien, den Pacatus schildert, die verbannung des Theodosius zu verstehen: dann bietet Pacatus eine schlagende bestätigung des Theodoret, denn dann spricht er von einem siege, den Theodosius über barbaren an der Donau erfocht unmittelbar nach seiner rilckkehr aus Spanien und zwar ehe er kaiser war, iam tum te tibi pugnare. Wenig trägt es aus, dass die chronisten von diesem siege schweigen. Sie stellen zusammen mit Orosius und Jordanis für diese zeit nur zwei, stellenweise sogar nur eine einzige überlieferung dar, indem sie sämmtlich ihre meisten nachrichten demselben sehr dürftigen wenn auch zuverlässigen annalenwerk entnehmen, dessen ursprüngliche fassung man in jedem gegebenen falle durch vergleichung der ableitungen wiederzugewinnen suchen muss. Auch der wichtige sieg des Modares 379 , durch den Thracien von den Gothen befreit wurde, ist uns nur in der wüsten anecdotenmasse des Zosimus erhalten, nicht bei den chronisten. Auf $Z_{0}$ simus selbst aber kann ein argumentum ex silentio gar nicht begründet werden, denn dieser theil seines werkes ist sehr ungeordnet. Vielleicht war auch der sieg des Theodosius an und für sich nicht grossartig und seine bedeutung lag melir in der moralischen wirkung. Nach alle dem sind wir meines erachtens nicht berechtigt, die angabe des Theodoret zu verwerfen, wir dürfen sie vielmehr durch Pacatus ergänzen und es ergiebt sich also: Gratian rief nach der niederlage des Valens bei Adrianopel 378 den Theodosius aus Spanien, wo er in einer art verbannung lebte, und gab ihm ein commando. Theodosius besiegte die Sarmaten und wurde am 19. januar 379 zu Sirmium in Illyrien von Gratian zum kaiser des ostens ernannt.

Dagegen ist es nicht wahrscheinlich, dass jener sieg über die Sarmaten diese erhebung veranlasste, wenigstens kann diese veranlassung nicht unmittelbar, nicht ausgesprochen gewesen sein, sonst würde Themistius in seiner begrüssungsrede dies unzweideutig hervortreten lassen. Theodoret sagt dies zwar, allein die anecdotenhafte form dieser behauptung zeigt hinreichend, dass sie nichts ist als ein geschwätz, wie es bedeutsame ereignisse regelmässig zu begleiten pflegt.

Göttingen.

Georg Kaufmann. 